\title{
LEGISLATION OF COMPETENCIES AND OPPORTUNITIES FOR SPECIALIZATION OF FELDSHERS AND PHYSICIAN ASSISTANTS IN PRIMARY HEALTH CARE
}

\author{
Penka Vracheva \\ Department of Health Care, Medical Faculty, Trakia University
}

\begin{abstract}
INTRODUCTION: The changing health needs of the population in terms of deficit and uneven distribution of medical staff, the increase in the cost of medical services and limited financial resources define the legislation for professional realization of feldshers and physician assistants as topical issue.
\end{abstract}

AIM: The aim of this paper is to explore:

- the legal regulation of the professional activities and competencies of feldshers and the possibilities for specialization with a focus on primary health care (PHC);

- the training of physician assistants for a career in PHC.

MATERIALS AND METHODS: Analysis of legal documents with regard to the professional realization and specialization of practicing feldschers and training of physician assistants was performed.

RESULTS AND DISCUSSION: The health care field professions Feldsher and Physician Assistant have different names but are characterized by similarity. The professionals are trained following a medical model, which differs from that for doctors by its accelerated pace and shorter time to acquire professional competencies and develop practical skills during the training. The competence of the professionals involves carrying out activities for overall care of patients by diagnosis, treatment and prevention. The certain degree of autonomy in their professional activities determines that feldshers and physician assistants are capable of supporting medical practices in PHC, mainly in small and remote locations. The analysis of the active regulatory documents determines the legislative regulation of: competencies and the opportunity for specialization in PHC for the practicing feldshers; training of physician assistants for a career in PHC. The conclusions of the study outline prospects for employment of feldschers and physician assistants in PHC.

Keywords: feldshers, physician assistants, PHC, competencies, professional activities

Address for correspondence:

Penka Vracheva

Department of Health Care

Medical Faculty

Trakia University

11 Armeyska St

6000 Stara Zagora

e-mail:p_vracheva@abv.bg

\section{INTRODUCTION}

The changing health needs of the population in terms of deficit and uneven distribution of medical staff, the increase in the cost of medical services and limited financial resources has created difficulties in servicing patients primarily in small and remote settlements. In these conditions, the idea of us- 
Legislation of Competencies and Opportunities for Specialization of Feldshers and Physician Assistants in Primary ...

ing professionals in primary health care (PHC), with delegated throughout the working process rights, is of current importance. Feldschers and the physician assistants who followed them are trained following a medical model which differs from the medical training for doctors by its accelerated pace and shorter time to acquire professional competencies and develop practical skills during the training. They are competent in performing activities for the overall care of the patients such as prophylactic, diagnostic and therapeutic care (4). This makes them professionals capable of assisting doctors, with certain rights of autonomous professional activities. The legislative regulation of the professional competencies of feldschers and physician assistants suggests the possibility of teamwork with physicians in PHC, in response to the growing health needs of the society.

\section{MATERIALS AND METHODS}

\section{Documentary Method}

Analysis of legal documents with regard to the professional realization and specialization of practicing feldshers and the training of physician assistants.

\section{RESULTS AND DISCUSSION}

After the start of the health reform in Bulgaria, as a result of legislative amendments to the Health Act (HA), Health Insurance Act (HIA), Medical Centers Act (MCA), Professional Organizations Act (POA), the feldshers were practicing without regulation, clear rules and legitimacy of their professional responsibilities. This is a serious challenge for those who are working in PHC. The uncertainty and dissatisfaction with job opportunities are a reason why some of the feldshers are leaving the health care system. With the announcement of Regulation 1 from 08.02.2011, which defines the professional activities of nurses, midwives and associated medical specialists, the activities of feldshers are legally regulated. In Article 7 and 8, Part IV of the Regulation there have been written different in volume and complexity activities on diagnosis, treatment and rehabilitation of patients, which the professionals can perform alone or after a doctor's assignment (4). These activities, defined as cognitive, analytico-synthetic and technical in ensuring full patient care, correspond to those in the professional standard competencies of staff with a professional qualification "Feldsher". Successful re- alization is possible in all structures, mostly outpatient care: centers and branches for emergency aid; in PHC; diagnostic consultative and medical centers; specialized dispensaries; centers for transfusion hematology; medico - social care centers for adults and the elderly, for children and adolescents; structures for medico - social care for persons with disabilities; hospice and palliative care structures; occupational health services; health insurance funds and organizations in the Ministry of Interior and the places of imprisonment; in the field of transport with the performance of pre-travel examinations; in the structures of the Bulgarian Red Cross $(3,4)$.

The professional realization of feldshers in PHC has its historical traditions. At present, general practitioners (GPs), who are practicing by the trading act, can choose to work in a team with professionals from the field of health care in the service of patients, who are registered with the medical practice. The preferences for feldshers are based on their medical training model, which allows the delegation of certain doctor rights and the performing of routine medical activities. By performing these activities, the feldshers complement and assist the medical practices (4). Under the conditions of incomplete health reform, feldshers are practicing in PHC only with legal relations with GPs $(1,4)$. However, this does not guarantee them a certain level of work security within the structure.

The current legislative regulation brings up the question of access to primary health care for populations in small and remote settlements, where there are no registered medical practices (1). The lack of such practices deprives the citizens of the opportunity to receive medical services, and of feldshers, who need to practice under doctor's supervision. Also, the absence of a legal possibility for direct contractual relationship of the feldshers with the National Health Insurance Fund (NHIF) to perform certain activities according to their competence. Nowadays, with the existing possibilities for communication, there are solutions which can meet the requirements for medical supervision of the professional activities of feldshers. This implies reserves and likely future changes in the legislation regarding the engagement and autonomous operation of feldshers and future physician assistants in PHC, with a contribution to improving the access of patients to primary care. The 
Penka Vracheva

opportunity for specialization of feldshers in primary health care under Regulation 1 of 01.22.2015 guarantees higher servicing quality, in response to the expectations of the patients (5).

The training of physician assistants started in 2014 in the Faculty of Public Health, Sofia and the Medical Faculty of the Trakia University in Stara Zagora. It still continues. The physician assistants appear to be followers of the feldshers, whose training was suspended in 1999. The training of the personnel in the Physician Assistant program with a professional qualification and educational qualification degree of Bachelor is full-time with workload of 4625 hours and acquisition of no less than 240 credits from the system of accumulation and transfer of credits $(6,7)$.

The working opportunities for physician assistants in PHC correspond to that of feldshers given the current regulations - Regulation 1; HA; professional standards for feldshers/physician assistants $(1,3,4)$. According to the Unified State Requirements (USR) for acquiring higher education, the Physician Assistant students undergo a modular training in primary care of 150 hours, clinical practice during the semester and pre-graduation internship in PHC. According to art. 17, p. 1 and 3 of USR, this provides them with sufficient knowledge and clinical experience to perform activities in the field of primary care $(6,8)$.

The analysis of the present regulations about the professional realization and specialization of practicing feldshers and the training of physician assistants is the basis for the formulation of these conclusions:

1. There is a legislative regulation of the professional activities and competencies for the professional realization of currently practicing feldschers.

2. There is opportunity for specialization of feldshers in PHC, which is guaranteed by a regulation.

3. The training of physician assistants provides them with good background and chance for successful realization in PHC.

4. The current situation and laws define the prospects for employment of feldschers and physician assistants in the PHC.

\section{REFERENCES}

1. Health Act (SG Issue 70 / 10.08.2004, last amend. and suppl. SG Issue 27 / 05.04.2016) (in Bulgarian)

2. Higher Education Act (SG Issue 112 / 27.12.1995, last amend. Issue 43 / 299.04.2008, the last suppl., Issue 42 / 05.06.2009) (in Bulgarian)

3. Professional Standards for the specialty "Feldscher"

4. Regulation 1 / 08.02.2011 on professional activities that nurses, midwives, associated medical specialists and healthcare assistants are able to perform under supervision or by themselves (SG /18.02.2011 Issue 15, and suppl. in SN №50/2011) (in Bulgarian)

5. Regulation $1 / 22.02 .2015$ on acquiring specialty in the healthcare system. SG. 2015; 7. (in Bulgarian)

6. Regulation of the Unified State Requirements for acquiring higher education in the specialties in vocational direction „Health Care“ (SG Issue 95 / 29.11.2005, title amend. and suppl. - SG Issue 32 of 2016) (in Bulgarian)

7. Regulation № 21 / 30.09.2004 for implementation of the system of accumulation and transfer of credits in higher education institutions. SG. 2004; 89.

8. Curriculum of the Physician Assistant program, Bachelor's degree 